\title{
VIOLENCIA ESCOLAR EN BACHILLERATO: ALGUNAS ESTRATEGIAS PARA SU PREVENCIÓN DESDE DIFERENTES PERSPECTIVAS
}

\author{
School violence in beccalaureate: some strategies for its \\ prevention from different perspectives
}

\author{
Mario Alberto GonzÁlez MedinA*, Diana Carolina TreviÑo VillarReAL*** \\ * Universidad de Monterrey. México \\ marioalberto.gonzalezm@udem.edu \\ https://orcid.org/0000-0002-8923-1491 \\ * Secretaría de Educación del Estado de Nuevo León. México. \\ diana.t.villarreal@gmail.com \\ bttp://orcid.org/0000-0002-2559-5368
}

Fecha de recepción: octubre de 2018

Fecha de aceptación: enero de 2019

\section{RESUMEN}

La violencia escolar es una acción que se ha venido manifestando a través de los años y esta ha afectado la vida de quienes la han padecido. Específicamente, el bachillerato es vulnerable a la violencia y va generando consecuencias negativas en los estudiantes, tanto en el ámbito escolar como en el personal. Es por ello que el objetivo del presente trabajo es determinar los factores que se relacionan con dicha violencia en estudiantes de este nivel, con la finalidad de contribuir a su prevención. El tipo de estudio fue no experimental, transversal, descriptivo y correlacional. La muestra estuvo conformada por 4.822 estudiantes. Se utilizaron los resultados de las Evaluaciones de Logro referidas al Sistema Educativo Nacional (ELSEN) y del cuestionario de contexto, ambos correspondientes al Plan Nacional para la Evaluación de los Aprendizajes (PLANEA) 2017. Se hizo una revisión exhaustiva de la literatura y de ahí se derivaron los factores propuestos en un modelo teórico. Se determinó la cantidad 
de alumnos que sufría de violencia escolar con base en su sexo y el tipo de escuela. Fueron aplicadas las pruebas t de student y la U de Mann-Whitney para evidenciar uno de los aspectos adversos que padecían los alumnos que experimentaban violencia escolar. Fue aplicada la técnica de modelaje con ecuaciones estructurales para verificar empíricamente el modelo teórico. Entre los hallazgos, se encontró una relación directa de la Violencia con los factores Emociones, Metas, Laboral y Relaciones, e indirecta con Actitud, Padres y Bienes. En conclusión, y tomando como base los resultados arrojados y las revisiones de diferentes autores, la violencia escolar está presente, no obstante, puede ser prevenida si se toman en cuenta los factores propuestos.

Palabras clave: violencia escolar; bachillerato; factores; modelo teórico; modelaje con ecuaciones estructurales.

\section{ABSTRACT}

School violence is an action that has been manifesting over the years and this has affected the lives of those who have suffered. Specifically, the baccalaureate is vulnerable to violence, and is generating negative consequences for students, both in the school setting and in the staff. That is why the objective of this paper is to determine the factors that are related to this violence in students of this level, with the purpose of contributing to its prevention. The type of study was non-experimental, cross-sectional, descriptive and correlational. The sample consisted of 4.822 students. The results of the Evaluaciones de Logro referidas al Sistema Educativo Nacional (ELSEN) and the context questionnaire were used, both corresponding to the Plan Nacional para la Evaluación de los Aprendizajes (PLANEA) 2017. There was a thorough review of the literature and from there the proposed factors were derived in a theoretical model. The number of students suffering from school violence was determined based on their sex and the type of school. Student t tests and the Mann-Whitney U test were applied to demonstrate one of the adverse aspects suffered by students who experienced school violence. The structural equations modeling technique was applied to empirically verify the theoretical model. Among the findings, a direct relationship was found between the Violence with the factors Emotions, Goals, Labor and Relationships, and indirect with Attitude, Parents and Assets. In conclusion, and based on the results and reviews of different authors, school violence is present, however, it can be prevented if the proposed factors are taken into account.

Key words: school violence; baccalaureate; factors; theoretical model; modeling with structural equations.

\section{INTRODUCCIÓN}

Es bien sabido que la violencia genera efectos negativos que conllevan a problemas futuros de diferente índole (Salcido Cibrián y Urías Aguirre, 2016), asimismo, y por desgracia, el ámbito escolar no está libre de ella, ya que es un acto que ha 
venido manifestándose por generaciones. De manera específica, en los adolescentes va forjando un desajuste psicosocial (Cava, Buelga, Musitu y Murgui, 2010), y a nivel escuela, inhibe acciones para contrarrestarla, llegando a ocultarse e incorporarse a la cultura de la institución (Rodríguez Figueroa, 2015), esto sin dejar de lado otras contrariedades que produce tras de sí. Al respecto, en el estado de Nuevo León, México, específicamente en el Plan Estatal de Desarrollo 2016-2021 del Programa Sectorial de Educación, ha quedado establecido que se deben realizar acciones para prevenir situaciones de riesgo, entre ellas, el acoso en el nivel de bachillerato (Gobierno del estado de Nuevo León, 2016); de ahí que resulta oportuno analizar los factores manifiestos que se relacionan con la violencia escolar, un objetivo que permitirá brindar pautas que orienten hacia cómo prevenirla, y así, alcanzar cambios efectivos, ya que, solo conociendo de cerca los antecedentes de los fenómenos educativos, se podrá reestructurar la escuela como institución promotora de una convivencia pacífica (Oliva, 2015), y por consiguiente, libre de violencia.

\section{ESTADO DE LA CUESTIÓN}

\subsection{Violencia en el contexto social}

Indiscutiblemente, la violencia es una grave violación a los derechos humanos (Organización Mundial de la Salud, 2014), y en esta predominan las relaciones de poder entre las personas involucradas. Frente a ello, existen expresiones tales como el autoritarismo, la opresión, la discriminación (Pérez García, 2016), entre otras.

En México, muchos niños y adolescentes se desarrollan en ambientes violentos de forma cotidiana (United Nations International Children's Emergency Fund, s.f.), lo cual, parte de una cultura de conflictos (Jiménez Ornelas, 2005), y en donde la escuela no escapa de su manifestación (García Lara y Cruz Pérez, 2018), de ahí la constante preocupación de padres y maestros (Bosworth, García, Judkins y Saliba, 2018). Ante esto, y puesto que la violencia escolar es el reflejo de la disgregación de la sociedad actual, no es posible abordar el tema de manera aislada sin antes generar vínculos que proyecten a la propia violencia como un tema prioritario para ser analizado, atendido y comprendido (Ayala Carillo, 2015), desde las estrategias que aborden distintos factores (Centers for Disease Control and Prevention, 2017).

\subsection{Violencia en el ámbito escolar}

La violencia escolar ha sido reconocida a nivel global como un problema serio y complejo (Menesini y Salmivalli, 2017), y esta es definida como aquella acción u omisión dolosa que tiene como propósito causar un daño físico o psicológico a cualquier individuo que pertenece a la comunidad educativa (Secretaría de Educación del Estado de Nuevo León, 2017); parte del uso intencional de la fuerza (Echeverri Ochoa, Gutiérrez García, Ramírez Sánchez y Morales Mesa, 2014) y se compone 
de muchos elementos para su caracterización (Prieto Quezada, Carrillo Navarro y Jiménez Mora, 2005). Este fenómeno no es nuevo, y es evidente que la sociedad no ha encontrado un camino eficaz para solucionarlo (Pérez García, 2016); en cuanto a la situación en México, su magnitud en algunos niveles educativos ha sido muy similar a la observada en otros países (Muñoz Abundez, 2008).

De manera concreta, el bachillerato ha sido calificado como vulnerable a la violencia (García Mandujano, 2015), y tan grave es el problema, que las escuelas han llegado a ser consideradas como instituciones productoras de esta (Toledo, Guajardo, Miranda y Pardo, 2018); viéndose involucrado el desempeño académico de los alumnos (del Tronco Paganelli y Madrigal Ramírez, 2013; García Lara y Cruz Pérez, 2018), las malas calificaciones (Zurita Ortega, Vilches Aznar, Martínez Martínez y Castro Sánchez, 2015), la reprobación de diversas asignaturas (Ruíz Ramírez, García Cué, Ruíz Martínez y Ruíz Martínez, 2018), entre otros. Con lo anterior, se deriva la pregunta ¿Qué factores están asociados con la violencia escolar?

\subsection{Relación emociones-metas y violencia escolar}

En efecto, las emociones y las metas están relacionadas entre sí, tal como se ha dado a conocer en un programa dirigido a alumnos de bachillerato y que es implementado por el Gobierno de México, en el cual se ha aseverado que existen ciertas emociones que ayudan y otras que impiden alcanzar las propias metas (Secretaría de Educación Pública y Programa de las Naciones Unidas para el Desarrollo, s.f.).

Por su parte, otros autores también han abordado el tema; Vitoria Hugo (2006) especificó que, al incidir sobre las emociones, probablemente se llegue también a las metas; García Retana (2012) concluyó que se debe revalorar el papel de las emociones en los procesos de aprendizaje, ya que son un elemento fundamental que impulsa a la acción, y es aquí en donde el logro de metas estaría involucrado; Aresté Grau (2015) afirmó que la educación de las emociones es imprescindible en la escuela y en la familia, y que la inteligencia emocional es elemental para alcanzar distintos tipos de metas, y Chiecher (2017) expuso que las emociones que despliegan los alumnos al resolver una tarea, son orientadas por el tipo de meta adoptada por ellos.

Ahora bien, tanto las emociones como las metas tienen influencia sobre la violencia; esta última es una expresión que está formada por acciones específicas, y en donde están incluidos valores y las propias emociones (Prieto Quezada et al., 2005). De ahí que Pacheco Salazar (2018) señalaba que los programas dirigidos a erradicarla y prevenirla en el ámbito escolar deberían considerar el trabajo desde la educación emocional. Asimismo, Sánchez, Ortega y Menesini (2012) abordaban que, para los agresores, la intervención debería focalizarse en la alfabetización emocional, y para las víctimas, la intervención debería canalizarse hacia el afrontamiento; y es aquí es en donde los docentes jugarían un papel fundamental al brindar apoyo para sobrellevar las emociones negativas (Hunter, Boyle y Warden, 2004). 
En estas afirmaciones, el trabajo de Mollá López, Prado Gascó y Martínez Rico (2015) es coincidente, ya que puntualizaron que, la capacidad que tienen los alumnos para reconocer y manejar correctamente las emociones pudiera ayudar a eliminar el acoso en las escuelas. Asimismo, Muñoz Prieto (2017) señaló que los estudiantes que poseen inteligencia emocional gestionarían sus sentimientos y los de los demás, y que este sería un recurso para actuar en las situaciones de violencia.

Posteriormente, y en lo que a las metas y violencia escolar se refiere, aquellas personas que logran sus metas a través de una mayor perseverancia, optimismo y confianza en sí mismas, regulan mejor sus emociones ante las manifestaciones de violencia (Quintana Peña, Montgomery y Malaver Soto, 2009). Al respecto, la Secretaría de Educación Pública (2017) en México, detallaba que resulta primordial que los estudiantes de bachillerato posean habilidades socioemocionales, mismas que empoderan para una mejor toma de decisiones y el logro de metas, y las cuales son necesarias en la prevención de conductas como la violencia y el acoso.

En este sentido, Bautista Gómez (2017) hablaba sobre la importancia de crear un programa educacional que tomara en cuenta la conceptualización de las metas de los alumnos, el desarrollo de ciertas estrategias para alcanzarlas y la motivación para la utilización de dichas estrategias, esto con el fin de inducir hacia un cambio conductual y emocional, e incidir de manera positiva en la eliminación de los actos violentos.

\subsection{Estudio de los padres, actividad laboral y relaciones educativas: factores ligados entre sí y su asociación con la violencia escolar}

En particular, la no violencia se construye principalmente a través de la educación (Narro Robles, Martuscelli Quintana y Barzana García, 2012); al respecto, y dado que la función de educar es una responsabilidad compleja, nada fácil, y a la que los padres se enfrentan, en muchas ocasiones con pocos recursos y desaciertos, es fundamental su propia educación, esto con el fin de brindar al hijo un desarrollo positivo e integral en todos los campos, incluyendo el escolar (Durán Gervilla et al., 2004); en cuanto a dicha tarea, y de no llevarse a cabo, pudiera conducir a lo abordado en el trabajo de Espitia Carrascal y Montes Rotela (2009), mismo que se realizó en un barrio de Colombia. Aquí, los padres no poseían condiciones para impulsar a sus hijos, esto es, sus prácticas, hábitos y recursos eran limitados, y es precisamente que, en la escuela, las quejas por parte de los maestros sobre la agresividad de los alumnos, la intolerancia y la violencia eran permanentes.

Esta última información coincide con Carneiro, Meghir y Parey (2013), quienes señalaron que el nivel de estudios de la madre está relacionado con el comportamiento de sus hijos. En otras palabras, los hijos que tienen mamás con un mayor nivel de estudios, muestran un mejor comportamiento.

Por añadidura, la baja escolarización de los padres genera otros aspectos perjudiciales, como las bajas expectativas escolares para con sus hijos; asimismo, en las 
MARIO ALBERTO GONZÁLEZ MEDINA Y DIANA CAROLINA TREVIÑO VILLARREAL VIOLENCIA ESCOLAR EN BACHILLERATO: ALGUNAS ESTRATEGIAS PARA SU PREVENCIÓN DESDE DIFERENTES PERSPECTIVAS

familias que cuentan con pocos recursos, es habitual observar que se privilegie el trabajo en lugar de la educación (Comisión Económica para América Latina y el Caribe (CEPAL) y Organización de las Naciones Unidas para la Educación, la Ciencia y la Cultura (UNESCO), 2009). Cabe destacar que la actividad laboral persiste debido a situaciones como las necesidades sin satisfacer (García y Chebez, 2009).

Particularmente, y para explicar mejor la situación del trabajo en México, según datos de la Encuesta Nacional de Ocupación y Empleo, en el año 2016 ocho de cada 100 niños y adolescentes trabajaban. Al respecto, el Módulo de Trabajo Infantil, a través de la Encuesta Nacional de Ocupación y Empleo puso en evidencia que, en el 2015, 8,4\% de los niños, niñas y adolescentes realizaban alguna actividad económica; de ellos, el 64,2\% tenía de 15 a 17 años (Instituto Nacional de Estadística y Geografía, 2017).

Por consiguiente, y como ya es conocido, realizar alguna actividad laboral en la infancia trae consigo cuestiones negativas a nivel personal (Oficina Internacional del Trabajo, 2014), esto sin contar los problemas a nivel escuela, entre ellos, de rendimiento académico, reprobación y analfabetismo (Secretaría del Trabajo y Previsión Social, 2014); pues igualmente, en estudiantes de bachillerato, el trabajo genera inconvenientes (Lorenzo, Ruiz Díaz, Toyos, Benitez y Santos, 2011), tal como lo hizo saber la Organización Internacional del Trabajo (2012), al hablar de que los lugares en donde los adolescentes realizan alguna actividad laboral pueden ocasionarles violencia verbal y física, y que esto estaría repercutiendo en otros trastornos; ahora bien, es posible que dichos actos se estarían trasladando a los espacios escolares.

De manera similar, Zurita Ortega et al. (2015) señalaban que la violencia, expresada en comportamientos agresivos y de victimización, se daba, pero entre aquellos adolescentes que pretendían insertarse en el mercado laboral; una acción que estaría generando malas relaciones en los planteles. Esta afirmación se fortalece con el trabajo de García (2006), quien señaló que los alumnos que trabajan, ven a la escuela como un sinónimo de imposición y menos como una institución placentera en donde se fortalecen las relaciones de socialización.

Ahora bien, si a esto se añade que en ocasiones las relaciones que se dan de forma cotidiana en las escuelas son rígidas y jerarquizan los grupos que la conforman, llegando a reproducir vínculos de poder e inseguridad entre sus miembros (Toledo et al., 2018), el problema se agudiza, por tanto, hay que considerar a las relaciones entre alumnos y docentes fundamentales, siendo estos últimos quienes deben adaptarse a las particularidades de los primeros para satisfacer sus necesidades en las cuestiones educativas y brindarles el tipo de ayuda que reclamen (Carbonero, Román, Martín Antón y Reoyo, 2009), incluyendo las preocupaciones ante los actos de violencia (Rodríguez Figueroa, 2015).

Aunado a esto, Volungis y Goodman (2017) hablaban sobre la importancia de la calidad de las relaciones entre alumnos y maestros, ya que esta pudiera jugar un papel significativo en la prevención de la violencia; Houdin Gómez (2013) recalcaba 
que la propia escuela requiere re-pensar su función, esto para transformarse en un espacio de protección para niños y adolescentes, en el cual puedan compartir acciones que fomenten relaciones de respeto, y Díaz Aguado (2005) señalaba que para prevenir la violencia desde las relaciones que se dan en la escuela es necesario redefinir los roles en los cuales se estructura la interacción educativa, además de brindar al alumno un papel activo en su educación, logrando que los profesores incrementen su autoridad y establezcan esquemas colaborativos en donde participen familia, escuela y sociedad.

En cuanto a la relación con la familia, sería importante que los alumnos mantuvieran buenas relaciones con ella, ya que Covarrubias Feregrino y Caro Luján (2016) afirmaron que cuando existe una mejor relación de los alumnos con los maestros, pero también con sus padres, hay una disminución en la probabilidad de que los propios alumnos cometan un mayor número de agresiones.

\subsection{Estudio de los padres y posesión de bienes; factores entrelazados en donde está involucrada la violencia escolar}

Retomando el factor estudio de los padres, este también se ha visto asociado con la posesión de bienes de los alumnos; Aguado Quintero, Girón Cruz y Salazar Silva (2006) señalaron que tanto la educación como la pobreza se retroalimentan; que las personas en situación de pobreza cuentan con pocos años de educación, probablemente por dicha situación, y viceversa, siendo la misma pobreza considerada desde diferentes aspectos enfocados en el consumo, ingreso y bienestar de las personas; algo similar fue reportado por Di Lorenzo y Rosales (2013) quienes relacionaron el no completar algún nivel educativo con la pobreza, y por Narro Robles y Moctezuma Navarro (2012), mismos que expusieron que el analfabetismo perdura en las familias que no cuentan con acceso a muchos bienes y servicios.

Por consiguiente, la violencia escolar se ha visto también relacionada con el factor bienes; Valdés Cuervo, Carlos Martínez y Torres Acuña (2012) señalaron que los problemas socioeconómicos y, por tanto, las limitaciones en la adquisición de bienes, son considerados como un factor que puede desencadenar la presencia de violencia escolar en adolescentes; Navarrete Noble (2016) dio a conocer que el contexto socioeconómico en el que viven los alumnos de bachillerato de cierta localidad, el cual es marcado por la violencia y la pobreza, refleja el tipo de relaciones que se ejercen dentro de la escuela, siendo la propia violencia un acto frecuente entre alumnos y con los maestros; Poujol Galván (2016) indicó que la violencia escolar y social guarda una dinámica compleja, en la cual está inmersa la escasez en bienes y desamparo; Cabezas Pizarro y Monge Rodríguez (2014) hicieron notar en un trabajo orientado a la influencia que tiene el entorno en donde se ubica la escuela para la manifestación del acoso en el salón de clases, que, un mayor porcentaje de dicho acoso es detectado en las zonas en donde predominan las necesidades insatisfechas de tipo económico y de bienes. Es por todo esto que 
sería imperioso realizar transformaciones profundas, como, por ejemplo, sacar de la pobreza extrema a muchos mexicanos (Furlán Malamud, 2016).

\subsection{Emociones y actitud personal; dos factores asociados con las relaciones educa- tivas y a la postre con la violencia escolar}

El factor emociones ya ha sido relacionado previamente con la violencia escolar, no obstante, este se ha visto asociado también con las relaciones educativas; dos factores que son indispensables para el desarrollo integral de las personas (Pérez Cabaní, Reyes Carretero y Juandó Bosch, 2001). Al respecto, Salcido Cibrián y Urías Aguirre (2016) expusieron que, incluir la educación de las emociones a través de cierto programa de inteligencia emocional, pudiera traer beneficios en las relaciones que se presentan en el ambiente escolar, la resolución y prevención de conflictos (en casos de violencia), entre otros. Una acción que resulta acertada si se toma en cuenta lo aportado por Vallés Arándiga (2014), quien expuso que las emociones son exteriorizadas por los alumnos, mismas que se expresan por las relaciones que tienen entre ellos y que generan diferentes tipos de violencia. De manera específica, Majata Álvarez, Flores Palacios y García Lara (2015) señalaban la presencia de emociones negativas (enojo y miedo) entre los estudiantes, esto tras las malas relaciones y actos de violencia escolar.

Por otro lado, las actitudes también han sido asociadas con las relaciones educativas y con la violencia escolar. Al respecto, dichas actitudes vienen a ser parte del perfil de las personas, y esto hace que tengan implicación en todas las acciones que se realizan (Romero Bojórquez, Utrilla Quiroz y Utrilla Quiroz, 2014), y un claro ejemplo de ello viene a ser la perseverancia, que es fundamental para los alumnos, ya que gracias a esta, los estudiantes terminan las tareas que comienzan y persiguen los objetivos que se trazan sin desanimarse (Instituto Nacional de Evaluación Educativa, 2014), por tanto, resulta significativo identificar dichas actitudes, así como también los rasgos del alumnado, ya que en los análisis sobre los determinantes del origen de la violencia y sus expresiones, esta última puede ser apreciada desde aquí (Rojas Bravo, 2013).

Otro aspecto que conforma la actitud personal es el estado de ánimo, siendo uno de los factores que se asocia con la violencia escolar. Esto es, la mayoría de los alumnos que tienen un estado de ánimo negativo, son víctima de violencia en la escuela (Continente, Giménez y Adell, 2010), una acción que se presenta tras las relaciones. Información similar a la de Lara Ros, Rodríguez Jiménez, Martínez González y Piqueras (2017), quienes señalaron que la depresión y ansiedad son características de las víctimas de violencia, y con la de Sierra Varón (2010), quien declaró que estos mismos niños se muestran ansiosos e inseguros, y que no logran concentrarse ni rendir en sus tareas escolares tras experimentar malas relaciones entre compañeros, o, al sufrir agresiones constantes. Del mismo modo, se ha abordado que los escolares que tienen una autopercepción negativa en el contexto escolar, 
muestran una tendencia de comportamiento violento (Martínez Ferrer, Murgui Pérez, Musitu Ochoa y Monreal Gimeno, 2008).

De frente a lo antedicho, se puede dar cuenta que los factores expuestos están relacionados con la violencia escolar, por tanto, a continuación se describirá el análisis para alcanzar el objetivo planteado, considerando las siguientes definiciones; Violencia, reflexionada como la actividad violenta expresada en cualquiera de sus formas y que se manifiesta dentro del contexto educativo (Secretaría de Educación Pública, s.f.); Metas, que no son más que el fin al que se direccionan las acciones (Real Academia Española, 2017a), y que regulan y organizan en los alumnos su comportamiento para la obtención de un logro (Alemán, Trías y Curione, 2011); Bienes, que hacen referencia a ciertas cosas que son idóneas para satisfacer algunas necesidades de las personas (Real Academia Española, 2017b), Padres, a los progenitores de los alumnos y a su nivel de estudios alcanzado; Actitud, a los aspectos de ánimo de los estudiantes como sus valores, y la tendencia al quehacer congruente con estos; resultando ser el potencial para la acción (Laca Arocena, 2005); Laboral, a la situación relacionada con el trabajo; Relaciones, a los vínculos existentes de estudiantes y docentes; y finalmente, Emociones, a las respuestas simples que tienen manifestaciones precisas, y las cuales vienen a convertirse en la exteriorización del reflejo que la persona siente ante alguna situación por enfrentar (Barragán Estrada y Morales Martínez, 2014).

\section{Método}

\subsection{Tipo de estudio}

El tipo de estudio fue no experimental, transversal, descriptivo y correlacional; no experimental ya que las variables no fueron manipuladas, esto es, solo se observaron (Hernández, Fernández y Baptista, 2006), transversal, ya que se estudió el fenómeno en un punto a través del tiempo, y descriptivo y correlacional, ya que se comprobó empíricamente el modelo teórico propuesto (García Salinero, 2004).

\subsection{Instrumentos}

Fueron utilizadas las bases de datos públicas del Instituto Nacional para la Evaluación de la Educación (INEE) en México, quien pone a disposición de la comunidad en general dicha información. Tales bases de datos contienen el resultado de los estudiantes de último grado de bachillerato en el estado de Nuevo León, México del ciclo escolar 2016-2017 en pruebas estandarizadas de las Evaluaciones de Logro referidas al Sistema Educativo Nacional (ELSEN) correspondiente al Plan Nacional para la Evaluación de los Aprendizajes (PLANEA) 2017. La evaluación ELSEN mide el logro del estudiante en las áreas de lenguaje y comunicación y de matemáticas por medio de 50 reactivos de opción múltiple en cada una de estas áreas. 
MARIO ALBERTO GONZÁLEZ MEDINA Y DIANA CAROLINA TREVIÑO VILLARREAL VIOLENCIA ESCOLAR EN BACHILLERATO: ALGUNAS ESTRATEGIAS PARA SU PREVENCIÓN DESDE DIFERENTES PERSPECTIVAS

Además, junto con el instrumento de evaluación de ELSEN, fue aplicado a los estudiantes un cuestionario de contexto que recogía de los jóvenes información concerniente a: 1) Datos generales, 2) Situación laboral, 3) Expectativas educativas, 4) Características personales, 5) Inglés y cómputo, 6) Trayectoria académica, 7) Entorno escolar, 8) Clima escolar y 9) Entorno familiar, entre otras cosas, esto los días 4 y 5 de abril del año 2017. De todas las preguntas que conforman los nueve aspectos mencionados, se seleccionaron aquellas que describieran a cada uno de los factores que se muestran en la tabla 1. En las preguntas (variables) del cuestionario se utilizó una escala Likert de 3 a 6 puntos dependiendo de la pregunta.

\subsection{Participantes}

La muestra estuvo compuesta por 4.822 estudiantes de bachillerato; 2.386 (49,5\%) hombres, 2.435 (50,5\%) mujeres y una respuesta omitida. La edad media y desviación estándar de los hombres fue de 18,0 años y 1,1 años, respectivamente, mientras que, para las mujeres, esos mismos estadísticos fueron de 17,8 años y 1,3 años, respectivamente. El total de alumnos pertenecientes a escuelas privadas fue de $657(13,6 \%)$ y $4.165(86,4 \%)$ de públicas. El tipo de muestreo fue estratificado; los estratos de donde se seleccionaron los alumnos de la muestra fueron los 32 estados de la República Mexicana. En dichos estados, las escuelas se clasificaron como medianas o grandes (más de 40 alumnos matriculados) y pequeñas ( 40 alumnos o menos). De las escuelas medianas o grandes, se seleccionó una muestra de estudiantes, mientras que, para las escuelas pequeñas, se tomaron a todos los alumnos como parte de la muestra (Instituto Nacional para la Evaluación de la Educación, 2018).

\subsection{Variables}

Para cumplir con el objetivo, previamente se hizo una revisión exhaustiva de la literatura, y de ella se propusieron los factores en los que, de alguna manera se pudiera tener injerencia para realizar cambios, y con ellos prevenir la Violencia escolar. Dichos factores se muestran en el modelo teórico (Figura 1) el cual expresa las relaciones entre los factores Laboral, Relaciones, Emociones, Metas, Bienes, Padres y Actitud con la Violencia escolar. El total de dichos factores fue de ocho y veintiséis variables medibles, y cada una de ellas fue medida a través de una pregunta o ítem. Todas las variables fueron de nivel ordinal y la descripción de las mismas en cada factor se detalla en la tabla 1. Todos los resultados fueron estadísticamente significativos para un p-valor $<0,05$. Los datos se procesaron en los softwares SPSS 25 y AMOS 25. 
Figura 1. Modelo teórico

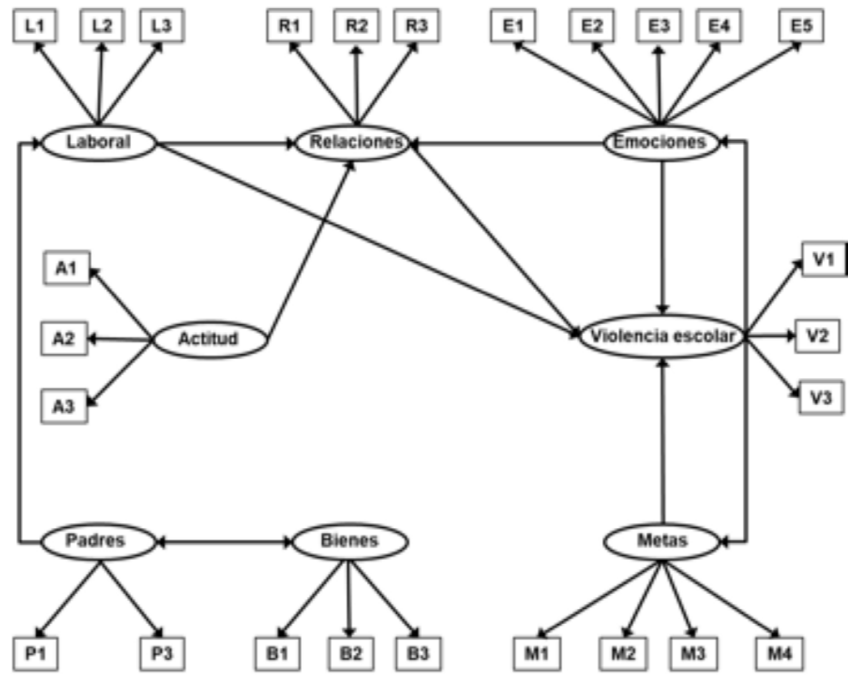

Fuente: Elabcracion propia con intormación de PLANEA 2017

Tabla 1. Factores y variables del modelo teórico

\begin{tabular}{|c|c|c|}
\hline Factor & $\begin{array}{c}\text { Variable } \\
\text { (Pregunta o ítem) }\end{array}$ & Valores \\
\hline $\begin{array}{l}\text { Violencia } \\
\text { escolar }\end{array}$ & $\begin{array}{l}\text { ¿Con qué frecuencias sufres de violencia? } \\
\text { - Física (V1). } \\
\text { - Verbal (V2). } \\
\text { - Robo (V3). }\end{array}$ & $\begin{array}{l}4=\text { Siempre } \\
3=\text { Frecuentemente } \\
2 \text { = Algunas veces } \\
1=\text { Nunca }\end{array}$ \\
\hline Metas & $\begin{array}{l}\text { - Alguna vez me obsesioné con una idea o meta } \\
\text { por un tiempo corto, pero más tarde perdí el } \\
\text { interés (M1). } \\
\text { - Tengo dificultad para mantener mi atención } \\
\text { en metas que requieren varios meses para ser } \\
\text { terminadas (M2). } \\
\text { - A menudo me pasa que después de tener una } \\
\text { meta cambio a otra (M3). } \\
\text { - A veces nuevas ideas o metas me distraen de } \\
\text { las que ya tenía establecidas (M4). }\end{array}$ & $\begin{array}{l}3=\text { Totalmente de } \\
\text { acuerdo } \\
2=\text { Parcialmente de } \\
\text { acuerdo } \\
1 \text { = Totalmente en } \\
\text { desacuerdo }\end{array}$ \\
\hline Bienes & $\begin{array}{l}\text { ¿Cuántos de los siguientes bienes hay en tu casa? } \\
\text { - Televisión (B2) } \\
\text { - Automóvil (B3) }\end{array}$ & $\begin{array}{l}5=\text { Cuatro } \\
4=\text { Tres } \\
3=\text { Dos } \\
2=\text { Uno } \\
1=\text { Ninguno }\end{array}$ \\
\hline
\end{tabular}


MARIO ALBERTO GONZÁLEZ MEDINA Y DIANA CAROLINA TREVIÑO VILLARREAL VIOLENCIA ESCOLAR EN BACHILLERATO: ALGUNAS ESTRATEGIAS PARA SU PREVENCIÓN DESDE DIFERENTES PERSPECTIVAS

\begin{tabular}{|c|c|c|}
\hline Factor & $\begin{array}{c}\text { Variable } \\
\text { (Pregunta o ítem) }\end{array}$ & Valores \\
\hline Padres & $\begin{array}{l}\text { ¿Cuál es el nivel educativo alcanzado por tu: } \\
\text { - madre? (P1) } \\
\text { - padre? (P2) }\end{array}$ & $\begin{array}{l}6=\text { Posgrado } \\
5=\text { Licenciatura } \\
4=\text { Bachillerato } \\
3=\text { Secundaria } \\
2=\text { Primaria } \\
1=\text { No estudió }\end{array}$ \\
\hline Actitud & $\begin{array}{l}\text { - Soy una persona que se esmera (A1) } \\
\text { - Termino todo lo que empiezo (A2) } \\
\text { - Soy una persona que trabaja duro (A3) }\end{array}$ & $\begin{array}{l}3=\text { Me describe } \\
\text { totalmente. } \\
2=\text { Me describe } \\
\text { parcialmente } \\
1=\text { No me describe }\end{array}$ \\
\hline \multirow{3}{*}{ Laboral } & - ¿Cuánto tiempo llevas trabajando? (L1) & $\begin{array}{l}6=\text { Más de } 2 \text { años } \\
5=\text { Entre } 1 \text { y } 2 \text { años } \\
4=\text { Entre } 6 \text { y } 11 \text { meses } \\
3=\text { Entre } 1 \text { y } 5 \text { meses } \\
2=\text { Menos de un mes } \\
1=\text { No trabajo }\end{array}$ \\
\hline & $\begin{array}{l}\text { - ¿Cuántas horas a la semana dedicas a trabajar? } \\
\text { (L2) }\end{array}$ & $\begin{array}{l}6=25 \text { o más } \\
5=\text { De } 19 \text { a } 24 \\
4=\text { De } 13 \text { a } 18 \\
3=\text { De } 7 \text { a } 12 \\
2=\text { De } 1 \text { a } 6 \\
1=\text { No trabajo }\end{array}$ \\
\hline & - ¿Trabajas actualmente? (L3) & $\begin{array}{l}3=\text { Tiempo completo } \\
2=\text { Medio tiempo } \\
1=\text { No trabajo }\end{array}$ \\
\hline Relaciones & $\begin{array}{l}\text { Califica las relaciones entre los miembros de la } \\
\text { comunidad escolar: } \\
\text { - Entre estudiantes (R1) } \\
\text { - Entre estudiantes y docentes (R2) } \\
\text { - Entre docentes (R3) } \\
\end{array}$ & $\begin{array}{l}4=\text { Excelente } \\
3=\text { Buena } \\
2=\text { Regular } \\
1=\text { Mala }\end{array}$ \\
\hline Emociones & $\begin{array}{l}\text { En el último mes, ¿con qué frecuencia te sucedió } \\
\text { lo siguiente? } \\
\text { - Me afectó algo que me ocurrió } \\
\text { inesperadamente (E1) } \\
\text { - Me sentí incapaz de controlar cosas } \\
\text { importantes en mi vida (E2) } \\
\text { - Pensé que no podía afrontar todas las cosas } \\
\text { que tenía que hacer (E3) } \\
\text { - Me enojé porque las cosas que me ocurrieron } \\
\text { estaban fuera de mi control (E4) } \\
\text { - Sentí que las dificultades se acumulaban tanto, } \\
\text { que no pude superarlas (E5) }\end{array}$ & $\begin{array}{l}4=\text { Siempre o casi } \\
\quad \text { siempre } \\
3=\text { Muchas veces } \\
2=\text { Pocas veces } \\
1=\text { Nunca o casi nunca }\end{array}$ \\
\hline
\end{tabular}

Fuente: Elaboración propia con información de PLANEA 2017 
MARIO ALBERTO GONZÁLEZ MEDINA Y DIANA CAROLINA TREVIÑO VILLARREAL VIOLENCIA ESCOLAR EN BACHILLERATO: ALGUNAS ESTRATEGIAS PARA SU PREVENCIÓN DESDE DIFERENTES PERSPECTIVAS

\subsection{Análisis de datos}

Como primer análisis, se determinó la cantidad de estudiantes con base en el sexo y el tipo de escuela (privada o pública) que sufrían de violencia escolar. Posteriormente, se aplicaron las pruebas t de student y la U de Mann-Whitney para poner en evidencia uno de los aspectos negativos que padecen los alumnos que experimentan violencia, esto al mostrar que existe una diferencia significativa en el resultado medio en las áreas de lenguaje y comunicación y de matemáticas entre los estudiantes con y sin violencia escolar por sexo y tipo de escuela.

Por último, se aplicó la técnica de modelaje con ecuaciones estructurales para verificar empíricamente el modelo teórico. Al tratarse de variables de nivel ordinal, el método de estimación fue el de mínimos cuadrados ponderados. Asimismo, se determinó el estadístico de bondad de ajuste Chi-cuadrada/grados de libertad = $\chi^{2} /$ g.l. Este cociente tiene que ser menor a 3 para ser admisible (Usluel, Aşkar y Baş, 2008); el índice absoluto Error de Aproximación Cuadrático Medio (RMSEA) que tiene que ser menor a 0,08 (Bagozzi y Youjae, 1988) y el índice predictivo, que es el Índice de Validación Cruzada Esperada (ECVI), siendo que su valor tiene que estar próximo a uno (Kline, 2005). Por otro lado, los índices incrementales o medidas de ajuste incremental, fueron el Índice Normado de Ajuste (NFI), que tiene que arrojar un resultado mayor a 0,9 para ser considerado como aceptable (Browne y Cudeck, 1993), el Índice de Tucker Lewis (TLI) con valores aprobados si el resultado es mayor a 0,9 (Jöreskog, 1969) y el Índice de Ajuste Normado de Parsimonia (PNFI) que tiene que acercarse a 1 (Lévy Mangin, 2003).

\section{Resultados}

La distribución de los alumnos que sufrían violencia escolar según el tipo de escuela y el sexo se muestra en la tabla 2. El porcentaje de escolares que sufrían violencia fue de 39,3\%; aproximadamente cuatro de cada diez alumnos vivían violencia escolar. De hecho, la distribución con base en el tipo de violencia que padecían se muestra en la tabla 3; el tipo de violencia más común fue la verbal (30,3\%).

Tabla 2. Distribución de alumnos que han sufrido violencia escolar

\begin{tabular}{|c|c|c|c|c|}
\hline \multirow{2}{*}{ Tipo de escuela } & Sexo & $\begin{array}{c}\text { No ha sufrido } \\
\text { violencia }\end{array}$ & $\begin{array}{c}\text { Sí ha sufrido } \\
\text { violencia }\end{array}$ & Total \\
\hline \multirow{2}{*}{ Pública } & Hombre & 1.125 & 948 & 2.073 \\
\cline { 2 - 5 } & Mujer & 1.365 & 697 & 2.062 \\
\hline \multirow{2}{*}{ Privada } & Hombre & 174 & 116 & 290 \\
\cline { 2 - 5 } & Mujer & 241 & 123 & 364 \\
\hline \multicolumn{2}{|r|}{ Total } & 2.905 & 1.884 & $4.789^{*}$ \\
\hline
\end{tabular}

*Los 33 datos faltantes para completar los 4.822 fueron valores perdidos.

Fuente: Elaboración propia con información de ELSEN 2017 
MARIO ALBERTO GONZÁLEZ MEDINA Y DIANA CAROLINA TREVIÑO VILLARREAL VIOLENCIA ESCOLAR EN BACHILLERATO: ALGUNAS ESTRATEGIAS PARA SU PREVENCIÓN DESDE DIFERENTES PERSPECTIVAS

Tabla 3. Distribución de alumnos por tipo de violencia escolar

\begin{tabular}{|c|c|c|c|}
\hline ¿Ha sufrido de violencia? & Física & Verbal & Robo \\
\hline No & $4.254(88,2 \%)$ & $3.322(68,9 \%)$ & $3.740(77,6 \%)$ \\
\hline Sí & $532(11,0 \%)$ & $1.463(30,3 \%)$ & $1.048(21,7 \%)$ \\
\hline Dato perdido & $36(0,8 \%)$ & $37(0,8 \%)$ & $34(0,7 \%)$ \\
\hline Total & 4.822 & 4.822 & 4.822 \\
\hline
\end{tabular}

Fuente: Elaboración propia con información de ELSEN 2017

Sin duda, la violencia escolar está presente en los estudiantes de bachillerato del estado de Nuevo León. En este sentido, la tabla 4 muestra los resultados en ambas áreas entre los estudiantes que sufrieron o no violencia escolar, clasificándolos por sexo y/o tipo de escuela. Los análisis estadísticos indican que, independientemente del sexo y/o tipo de escuela, los alumnos que sufrían violencia escolar obtuvieron en promedio resultados significativamente inferiores en cada área que sus pares que no padecían de violencia escolar. En otras palabras, experimentar violencia escolar se asoció con obtener resultados inferiores en ambas áreas. Ahora, se determinan los factores que se asocian al fenómeno descrito. Dichas relaciones se obtuvieron aplicando la técnica de modelaje con ecuaciones estructurales, cuyos resultados se muestran en la figura 2.

Tabla 4. Comparación de los resultados de lenguaje y comunicación (LYC) y de matemáticas (MAT)

\begin{tabular}{|c|c|c|c|c|c|c|c|c|}
\hline & \multicolumn{5}{|c|}{ Mujer } & \multicolumn{5}{c|}{ Hombre } \\
\hline Área & CV & SV & Estadístico & p-valor & CV & SV & Estadístico & p-valor \\
\hline LYC & 506,6 & 529,4 & $\mathrm{Z}=-5,080$ & 0,000 & 488,3 & 502,1 & $\mathrm{Z}=-2,968$ & 0,003 \\
\hline MAT & 484,7 & 509,0 & $\mathrm{Z}=-4,827$ & 0,000 & 513,1 & 527 & $\mathrm{Z}=-2,083$ & 0,037 \\
\hline & \multicolumn{6}{|c|}{ Privada } & \multicolumn{5}{c|}{ Pública } \\
\hline Área & CV & SV & Estadístico & p-valor & CV & SV & Estadístico & p-valor \\
\hline LYC & 489,6 & 524,4 & $\mathrm{Z}=-3,818$ & 0,000 & 497,2 & 516,1 & $\mathrm{Z}=-5,54$ & 0,000 \\
\hline MAT & 486,2 & 530,3 & $\mathrm{Z}=-4,242$ & 0,000 & 502,8 & 514,8 & $\mathrm{Z}=-2,66$ & 0,008 \\
\hline
\end{tabular}

\begin{tabular}{|c|c|c|c|c|c|c|c|c|}
\hline \multicolumn{9}{|c|}{ Privada } \\
\hline & \multicolumn{9}{|c|}{ Mujer } & \multicolumn{4}{c|}{ Hombre } \\
\hline Área & CV & SV & Estadístico & p-valor & CV & SV & Estadístico & p-valor \\
\hline LYC & 495,8 & 523,3 & $\mathrm{Z}=-2,461$ & 0,014 & 483,1 & 525,9 & $\mathrm{t}=3,235$ & 0,001 \\
\hline MAT & 466,4 & 510,2 & $\mathrm{Z}=-3,263$ & 0,001 & 507,2 & 558,1 & $\mathrm{Z}=-3,366$ & 0,001 \\
\hline
\end{tabular}


MARIO ALBERTO GONZÁLEZ MEDINA Y DIANA CAROLINA TREVIÑO VILLARREAL VIOLENCIA ESCOLAR EN BACHILLERATO: ALGUNAS ESTRATEGIAS PARA SU PREVENCIÓN DESDE DIFERENTES PERSPECTIVAS

\begin{tabular}{|c|c|c|c|c|c|c|c|c|}
\hline \multicolumn{9}{|c|}{ Pública } \\
\hline & \multicolumn{9}{|c|}{ Mujer } & \multicolumn{5}{c|}{ Hombre } \\
\hline Área & CV & SV & Estadístico & p-valor & CV & SV & Estadístico & p-valor \\
\hline LYC & 508,5 & 530,5 & $\mathrm{t}=4,714$ & 0,000 & 488,9 & 498,4 & $\mathrm{Z}=-1,989$ & 0,042 \\
\hline MAT & 487,9 & 508,8 & $\mathrm{Z}=-3,861$ & 0,000 & 501,8 & 522,2 & $\mathrm{Z}=-2,432$ & 0,006 \\
\hline
\end{tabular}

Z: Se aplicó la prueba no paramétrica U de Mann-Whitney.

t: Se aplicó la prueba paramétrica de t de student.

$\mathrm{CV}$ : Con violencia.

SV: Sin violencia.

Fuente: Elaboración propia con información de ELSEN 2017

Figura 2. Modelo empírico

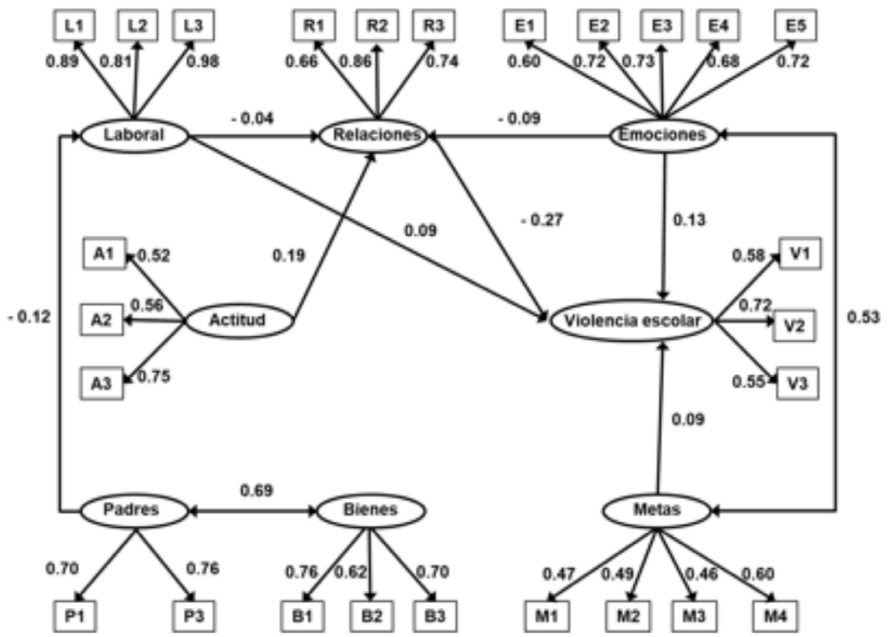

Estadísticos de bondad de ajuste: Chi-cuadrada (1731.949)/grados de libertad $=\chi^{2} / g . l .=1.993$ $<3$ (Usluel et al., 2008), el Error de Aproximación Cuadrático Medio (RMSEA) $=0.0 .032$ cuyo intervalo de confianza del 90\% fue $(0.031,0.034)$, el Índice de Validación Esperada (ECVI) = 0.396, el Índice Normado de Ajuste $(\mathrm{NFI})=0.0 .954$, el Índice de Tucker Lewis (TLI) $=0.953$. La medida de parsimonia fue el Índice de Ajuste Normado de Parsimonia $(\mathrm{PNFI})=0.786$. El p-valor del modelo general fue de 0.000. Sin embargo, dado el número de grados de libertad este último valor no es confiable (Acock, 2013). Las asociaciones entre los factores fueron significativas (Escobedo Portillo, Hernández Gómez, Estebané Ortega y Martínez Moreno, 2016).

Fuente: Elaboración propia 
El modelo empírico (Figura 2) arrojó relaciones directas e indirectas entre los factores propuestos y la Violencia escolar. El factor Emociones fue el de mayor peso $(0,13)$ en la Violencia escolar, es decir, un estudiante que no pueda afrontar las cosas que tiene que hacer, sea incapaz de controlar las cosas importantes, sienta que las dificultades se acumulan y no las puede superar, se enoje porque las cosas que ocurren están fuera de su control, y que le afecte lo que ocurre inesperadamente, está asociado con un incremento de la Violencia escolar.

El siguiente factor fue Metas, con un peso de 0,09. Esto es, un alumno que, en ocasiones nuevas ideas o metas lo distraigan de las que ya tenía, que tenga dificultad para mantener su atención en metas que requieren varios meses para ser terminadas, que a veces se obsesione con una idea o meta por un tiempo corto, pero más tarde pierda el interés, y que frecuentemente después de tener una meta cambie a otra, se relaciona con un aumento en la Violencia escolar. Por su parte, el factor Laboral también tuvo un peso de 0,09 en la Violencia, es decir, trabajar por más tiempo se relaciona con padecer de violencia escolar.

En cuanto al último factor que se relacionó de manera directa con la Violencia fue Relaciones con un peso de - 0,27. Es decir, a medida que las relaciones entre estudiantes con sus pares y/o docentes mejoran, la Violencia escolar baja.

De forma indirecta, los factores Emociones y Laboral tuvieron un peso de 0,024 y 0,010 , respectivamente con la Violencia. En este caso, mejorar los aspectos que componen las Emociones y una disminución en la actividad laboral se relacionó con una baja de la Violencia escolar. Por su parte, Actitud y Padres tuvieron un peso indirecto de - 0,051 y - 0,010, respectivamente con la Violencia. Es decir, ser un estudiante que se esmera, que termina todo lo que comienza, que trabaja duro y que tiene padres con un nivel de estudios más alto, se asocia con una disminución de la Violencia escolar.

Los factores que presentaron una relación directa con el factor Relaciones fueron Emociones, Actitud y Laboral con un peso - 0,09, 0,19 y - 0,04, respectivamente. Esto es, tener un mejor control de las variables que conforman las Emociones, una Actitud positiva y disminuir la parte Laboral, se asocia con un clima positivo en las Relaciones entre los estudiantes y/o docentes. Por último, los factores Padres y Bienes tuvieron una correlación positiva de 0,69; mientras los padres tengan un nivel de estudios más alto, sus hijos tendrán una mayor cantidad de bienes.

Por otra parte, las Emociones y Metas tuvieron una correlación de 0,53, esto es, un estudiante que le afecte algo que le ocurre de forma inesperada, se sienta incapaz de controlar las cosas importantes en su vida, no pueda afrontar todas las cosas que tenga que hacer, se moleste porque las cosas que ocurren están fuera de su control y sienta que las dificultades se acumulan a un punto que no las puede superar, se asocia con obsesionarse con una meta por un corto tiempo y luego perder el interés en ella, tener dificultad en mantener su atención en metas que requieren de varios meses para ser terminadas, con el cambio de metas y distraerse con nuevas ideas o metas. 
MARIO ALBERTO GONZÁLEZ MEDINA Y DIANA CAROLINA TREVIÑO VILLARREAL VIOLENCIA ESCOLAR EN BACHILLERATO: ALGUNAS ESTRATEGIAS PARA SU PREVENCIÓN DESDE DIFERENTES PERSPECTIVAS

\section{CONCLUSIONES Y DISCUSIÓN}

De acuerdo con lo expuesto, la violencia escolar es un acto que está presente en los planteles de bachillerato en Nuevo León, México, y esta ha traído resultados significativamente bajos en las áreas de lenguaje y comunicación y de matemáticas en los estudiantes que la padecen, información que coincide con otro trabajo en donde se afirmaba que, los alumnos que experimentan violencia, obtienen puntajes inferiores en el logro en las áreas de lenguaje y comunicación y de matemáticas (González Medina y Treviño Villarreal, 2018). Por lo anterior, se darán a conocer las pautas para la prevención de la violencia escolar.

El factor Emociones se relacionó de manera directa con la Violencia escolar, y así lo hicieron saber también Mollá López et al. (2015) y Muñoz Prieto (2017), por ello, es prioritario promover las siguientes acciones entre los alumnos: a) el que afronten sus responsabilidades escolares b) sean competentes para controlar los sucesos trascendentales, c) eviten el acumular dificultades, y si las presentan, que las puedan superar, d) no molestarse porque las cosas que les sucedan se salgan de control, y e) evitar que les aqueje aquello que les ocurra de manera inesperada. No obstante, para alcanzar lo ya señalado, resulta una necesidad que los programas orientados a erradicar y prevenir la violencia, consideren el trabajar bajo la línea de la educación emocional, tal como lo afirmó Pacheco Salazar (2018), pero tomando en cuenta las diferencias entre agresores y víctimas (Sánchez, Ortega y Menesini, 2012).

Otro factor relacionado directamente con la Violencia escolar fue Metas, una evidencia afín a lo señalado por Quintana Peña et al. (2009), por tanto, hay que impedir en los estudiantes de bachillerato que: a) nuevas ideas los distraigan de las que tenían inicialmente, b) se obsesionen con una idea o meta por un periodo corto y después, pierdan el interés, y c) cambien constantemente de una meta a otra. Por el contrario, hay que establecer estrategias para que sepan mantener su atención en aquellas metas que requieren cierto tiempo para ser concluidas. Asimismo, es necesaria la creación de un programa educativo que tome en cuenta las metas del alumnado, esto para incidir positivamente en la erradicación de las acciones violentas, tal como lo expuso Bautista Gómez (2017).

En cuanto al factor Laboral, este también tuvo una relación positiva con la Violencia escolar de modo directo, una aseveración semejante a la de Zurita Ortega et al. (2015) y a la descrita por la Organización Internacional del Trabajo (2012); por tanto, y ante los casos manifiestos en los cuales el alumnado lleve a cabo una actividad laboral, valdría la pena promover que esta se realice por un menor tiempo.

El factor Relaciones tuvo una asociación directa con la Violencia escolar, información afín a la de Volungis y Goodman (2017) y a la de Covarrubias et al. (2016). Derivado de esto, resulta trascendente que el trato que se dé entre alumnos y maestros mejore, además, sería importante que los docentes estén atentos a las preocupaciones existentes relacionadas con la violencia (Rodríguez Figueroa, 2015). 
MARIO ALBERTO GONZÁLEZ MEDINA Y DIANA CAROLINA TREVIÑO VILLARREAL VIOLENCIA ESCOLAR EN BACHILLERATO: ALGUNAS ESTRATEGIAS PARA SU PREVENCIÓN DESDE DIFERENTES PERSPECTIVAS

Con referencia al factor Actitud, este tuvo una relación indirecta y negativa con la Violencia escolar. Es decir, a medida que la Actitud disminuye, la Violencia escolar aumenta. Información similar a la de Rojas Bravo (2013), Continente et al. (2010) y Lara Ros et al. (2017); por tanto, es importante fomentar en los alumnos: a) el esmero al realizar sus actividades, b) la conclusión de todo lo que emprendan, y c) el trabajo duro, con esto, se puede mejorar el factor Actitud y a su vez disminuir la violencia.

En cuanto al factor Padres, este también tuvo una relación indirecta con la Violencia escolar, lo que coincide con Espitia Carrascal y Montes Rotela (2009). Por ello, valdría la pena promover la educación para padres, tal como lo dio a conocer Durán Gervilla, et al. (2004), ya que se ha observado que el nivel de estudios de la mamá, se relaciona con un mejor comportamiento en los hijos (Carneiro et al., 2013). Cabe resaltar que este acto, además, pudiera traer consigo otros beneficios, como mejorar los resultados de los alumnos en algunas asignaturas (González Medina y Treviño Villarreal, 2018).

También hubo asociaciones de los factores Emociones con Relaciones y posteriormente con la Violencia escolar, así como lo dieron a conocer Vallés Arándiga (2014) y Majata Álvarez et al. (2015), por tanto, resulta pertinente lo abordado por Salcido Cibrián y Urías Aguirre (2016) sobre la idea de establecer un programa en donde se incluya la educación de las emociones, lo cual traería beneficios en las relaciones escolares, además de prevenir conflictos.

Otras asociaciones que se dieron fueron entre los factores Actitud, Relaciones y Violencia escolar y Laboral, Relaciones y Violencia escolar, una manifestación semejante a la descrita por Sierra Varón (2010) y García (2006), respectivamente.

Subsecuentemente, el factor Padres estuvo relacionado de manera directa con el factor Bienes, al igual que lo reportaron Aguado Quintero et al. (2006), Di Lorenzo y Rosales (2013) y Narro Robles y Moctezuma Navarro (2012). Lo dicho resulta hasta cierto punto nomotético, ya que, cuando los progenitores cuentan con un nivel de estudios más elevado, pudieran estar aspirando a mejores empleos, por consiguiente, contarían con remuneraciones más altas y estarían adquiriendo así una mayor cantidad de bienes, mismos que quedarían al alcance y para beneficio de los hijos.

En concreto, y al volver la mirada hacia lo expuesto, claramente se puede observar que la violencia escolar en bachillerato puede ser prevenida; para ello, es necesaria la intervención de los actores que están involucrados en la práctica educativa. Sin duda, los investigadores cobran especial relevancia, ya que son ellos quienes permitirán dar cuenta de otros factores que estén asociados con la violencia escolar. Por lo pronto, este estudio sirve de parteaguas para nuevos trabajos que aborden dicha temática, la cual es crucial en nuestros días. 
MARIO ALBERTO GONZÁLEZ MEDINA Y DIANA CAROLINA TREVIÑO VILLARREAL VIOLENCIA ESCOLAR EN BACHILLERATO: ALGUNAS ESTRATEGIAS PARA SU PREVENCIÓN DESDE DIFERENTES PERSPECTIVAS

\section{REFERENCIAS BIBLIOGRÁFICAS}

Acock, A. (2013). Discovering Structural Equation Modeling Using Stata. Texas: College Station.

Aguado Quintero, L. F., Girón Cruz, L. E. y Salazar Silva, F. (2006). Relación entre pobreza y educación urbanas en el Valle del Cauca. Revista Latinoamericana de Desarrollo Económico, 7. http://www.scielo.org.bo/scielo.php?script=sci_arttext\&pid=S2074-47062006000200003 (Consultado el 8 de enero de 2019).

Alemán, M. J., Trías, D. y Curione, K. (2011). Orientaciones motivacionales, rendimiento académico y género en estudiantes de bachillerato. Ciencias Psicológicas, 5(2). http://www. scielo.edu.uy/scielo.php?script=sci_arttext\&pid=S1688-42212011000200004 (Consultado el 1 de octubre de 2018).

Aresté Grau, J. (2015). Las emociones en educación infantil: sentir, reconocer y expresar. Logroño: Universidad Internacional de la Rioja. https://reunir.unir.net/bitstream/ handle/123456789/3212/ARESTE\%20GRAU\%2C\%20JUDIT.pdf?sequence=1 (Consultado el 9 de enero de 2019).

Barragán Estrada, A. R. y Morales Martínez, C. I. (2014). Psicología de las emociones positivas: generalidades y beneficios. Enseñanza e Investigación en Psicología, 19(1), 103-118.

Bautista Gómez, L. M. (2017). Actitud emprendedora y proyectos de vida en contexto vulnerable: Un acercamiento con estudiantes de secundaria en San Juan de la Maguana. Santo Domingo: Universitat de València, Servei de Biblioteques i Documentació. http://roderic.uv.es/bitstream/handle/10550/61137/TESIS\%20LESIA\%20roderic. pdf?sequence=1\&isAllowed=y (Consultado el 6 de octubre de 2018).

Bagozzi, R. P. y Youjae, Y. (1988). On the evaluation of Structural Equation models. Journal of the Academy of Marketing Science, 16(1), 74-94. http://dx.doi.org/10.1007/BF02723327

Bosworth, K.. García, R.. Judkins, M. y Saliba, M. (2018). The impact of leadership involvement in enhancing high school climate and reducing bullying: An exploratory study. Journal of School Violence. 17(3), 354-366. https://doi.org/10.1080/15388220.2017.1376208

Browne, M. W. y Cudeck, R. (1993). Alternative ways of assessing model fit. Thousand Oaks: Sage Publications.

Cabezas Pizarro, H. y Monge Rodríguez, M. (2014). Influencia del entorno donde se ubica el centro educativo en la presencia del acoso en el aula. Revista Actualidades Investigativas en Educación, 14(3), 1-22.

Carbonero, M. A., Román, J. M., Martín Antón, L. J. y Reoyo, N. (2009). Efecto del programa de habilidades docentes motivadoras en el profesorado de secundaria. Revista de Psicodidáctica, 14 (2), 229-244.

Carneiro, P., Meghir, C. y Parey, M. (2013). Maternal education, home environments, and the development of children and adolescents. Journal of the European Economic Association, 11(1), 123-160. https://doi.org/10.1111/j.1542-4774.2012.01096.x

Cava, M. J., Buelga, S., Musitu, G. y Murgui, S. (2010). Violencia escolar entre adolescentes y sus implicaciones en el ajuste psicosocial: un estudio longitudinal. Revista de Psicodidáctica, 15(1), 21-34.

Centers for Disease Control and Prevention (2017). School violence: Prevention. Atlanta: Centers for Disease Control and Prevention. 
Comisión Económica para América Latina y el Caribe (CEPAL) y Organización de las Naciones Unidas para la Educación, la Ciencia y la Cultura (UNESCO) (2009). Impacto social y económico del analfabetismo: modelo de análisis y estudio piloto. Nueva York: CEPAL/ UNESCO. https://www.cepal.org/es/publicaciones/3747-impacto-social-economicoanalfabetismo-modelo-analisis-estudio-piloto (Consultado el 8 de enero de 2019).

Continente, X. G., Giménez, A. P. y Adell, M. N. (2010). Factores relacionados con el acoso escolar (bullying) en los adolescentes de Barcelona. Gaceta Sanitaria, 24(2), 103-108.

Covarrubias Feregrino, A. y Caro Luján, N. R. (2016). Determinantes de la violencia entre pares en escuelas secundarias del Estado de México: calidad de relaciones con maestros y familiares. RIDE. Revista Iberoamericana para la Investigación y el Desarrollo Educativo, 7(13). http://www.scielo.org.mx/scielo.php?script=sci_arttext\&pid=S2007-74672016000200220 (Consultado el 20 de septiembre de 2018).

Chiecher, A. C. (2017). Metas y contextos de aprendizaje. Un estudio con alumnos del primer año de carreras de ingeniería. Innovación Educativa, 17(74), 61-80.

Del Tronco Paganelli, J. y Madrigal Ramírez, A. (2013). Violencia escolar en México: una exploración de sus dimensiones y consecuencias. Revista Trabajo Social UNAM, 4, 23-42. http://www.revistas.unam.mx/index.php/ents/article/viewFile/54048/48108 (Consultado el 10 de agosto de 2018).

Di Lorenzo, L. N. y Rosales, G. A. (2013). Analfabetismo y pobreza en San Luis: un estudio descriptivo y comparativo. Fundamentos en Humanidades, 14(28), 29-49.

Díaz Aguado, M. J. (2005). Por qué se produce la violencia escolar y cómo prevenirla. Revista Iberoamericana de Educación, 37. https://rieoei.org/historico/documentos/rie37a01. htm (Consultado el 18 de septiembre de 2018).

Durán Gervilla, A., Tébar Pérez, M. D., Ochando Korhonen, B., Martí Chiva, M. A., Bueno Cañigral, F. J., Pin Arboledas, G., Cubel Alarcón, M. M. y Genís Giner, M. R. (2004). Manual didáctico para la escuela de padres. Valencia: Fundación para el Estudio, Prevención y Asistencia a las Drogodependencias. https://www.navarra.es/NR/rdonlyres/7DEF9FD2CC0B-4B2F-86A5-E218D9DD672C/243833/Manualdidacticopadresymadres.pdf (Consultado el 19 de septiembre de 2018).

Echeverri Ochoa, A., Gutiérrez García, R. A., Ramírez Sánchez, C. M. y Morales Mesa, S. A. (2014). Hacia una construcción del concepto violencias escolares. Revista Colombiana de Ciencias Sociales, 5(1), 122-138.

Escobedo Portillo, M. T., Hernández Gómez, J. A., Estebané Ortega, V. y Martínez Moreno, G. (2016). Modelos de ecuaciones estructurales: características, fases, construcción, aplicación y resultados. Ciencia \& Trabajo, 18(55). http://dx.doi.org/10.4067/S071824492016000100004

Espitia Carrascal, R. E. y Montes Rotela, M. (2009). Influencia de la familia en el proceso educativo de los menores del barrio Costa Azul de Sincelejo (Colombia). Investigación y Desarrollo, 17(1), 84-105.

Furlán Malamud, A. (2016). Inseguridad y violencia en la educación. Problemas y alternativas. Perfiles Educativos, 34, 118-128.

García, M. L. (2006). Trabajo infantil y experiencia escolar. Buenos Aires: Instituto Internacional de Planeamiento de la Educación/Organización de las Naciones Unidas para la Educación, la Ciencia y la Cultura. 
MARIO ALBERTO GONZÁLEZ MEDINA Y DIANA CAROLINA TREVIÑO VILLARREAL VIOLENCIA ESCOLAR EN BACHILLERATO: ALGUNAS ESTRATEGIAS PARA SU PREVENCIÓN DESDE DIFERENTES PERSPECTIVAS

García, M. L. y Chebez, V. (2009). ¿Qué hacemos con el trabajo infantil desde la escuela?: material de capacitación docente. Buenos Aires: Organización de las Naciones Unidas para la Educación, la Ciencia y la Cultura/Instituto Internacional de Planeamiento de la Educación.

García Lara, G. A. y Cruz Pérez, O. (2018). Sociedad y violencia: sujetos, prácticas y discursos. Manual Moderno.

García Mandujano, M. A. (2015). Violencia y acoso escolar: descripción y tipos de adolescentes que participan en contextos educativos de nivel media superior. México: Universidad Autónoma del Estado de México. http://ri.uaemex.mx/handle/20.500.11799/49965 (Consultado el 12 de septiembre de 2018).

García Retana, J. A. (2012). La educación emocional, su importancia en el proceso de aprendizaje. Educación, 36(1), 1-24.

García Salinero, J. (2004). Estudios descriptivos. Nure Investigación, 7. http://webpersonal. uma.es/de/jmpaez/websci/BLOQUEIII/DocbIII/Estudios\%20descriptivos.pdf (Consultado el 24 de octubre de 2018).

Gobierno del Estado de Nuevo León (2016). Plan Estatal de Desarrollo 2016-2021. Programa Sectorial de Educación. Monterrey, Gobierno del estado de Nuevo León. http://www. nl.gob.mx/publicaciones/plan-estatal-de-desarrollo-2016-2021 (Consultado el 28 de septiembre de 2018).

González Medina, M. A. y Treviño Villarreal, D. C. (2018). Logro educativo y factores asociados en estudiantes de sexto grado de educación primaria en el estado de Nuevo León, México. Perfiles Educativos, 40(159), 107-125.

Hernández, R., Fernández, C. y Baptista, P. (2006). Metodología de la investigación. México: Mc Graw Hill.

Houdin Gómez, C. (2013). Violencia en las instituciones educativas. Un problema complejo. Revista Integra Educativa, 6(2). http://www.scielo.org.bo/scielo.php?script=sci_arttext \&pid=\$1997-40432013000200006 (Consultado el 19 de septiembre de 2018).

Hunter, S. C., Boyle, J. M. E. y Warden, D. (2004). Help seeking amongst child and adolescent victims of peer-aggression and bullying: the influence of school-stage, gender, victimization, appraisal, and emotion. British Journal of Educational Psychology, 74(3), 375-390. https://doi.org/10.1348/0007099041552378

Instituto Nacional de Estadística y Geografía (2017). Estadísticas a propósito del día del niño (30 de abril). Datos nacionales. Aguascalientes: Instituto Nacional de Estadística y Geografía. http://www.inegi.org.mx/saladeprensa/aproposito/2017/ni\%C3\%B1o2017_Nal. pdf (Consultado el 15 de noviembre de 2018).

Instituto Nacional de Evaluación Educativa (2014). Perseverando hacia el éxito en los estudios: PISA 2012 y las CC.AA. Madrid: Ministerio de Educación, Cultura y Deporte. http://www. mecd.gob.es/inee/dam/jcr:d0e8d69e-66e1-4812-8501-3670dc7c864f/educaineeautonomias3.pdf (Consultado el 26 de septiembre de 2018).

Instituto Nacional para la Evaluación de la Educación (2018). Panorama educativo de México 2017. Indicadores del Sistema Educativo Nacional. Educación básica y media superior. México: Instituto Nacional para la Evaluación de la Educación. 
MARIO ALBERTO GONZÁLEZ MEDINA Y DIANA CAROLINA TREVIÑO VILLARREAL VIOLENCIA ESCOLAR EN BACHILLERATO: ALGUNAS ESTRATEGIAS PARA SU PREVENCIÓN DESDE DIFERENTES PERSPECTIVAS

Jiménez Ornelas, R. A. (2005). La delincuencia juvenil: fenómeno de la sociedad actual. Papeles de Población, 11(43), 215-261.

Jöreskog K. G. (1969). A general approach to confirmatory maximum likelihood factor analysis. Psychometrika, 34 (2), 183-202. https://doi.org/10.1007/BF02289343

Kline R. B. (2005). Principles and practice of structural equation modeling. Nueva York: Guilford Press. (Consultado el 9 de enero de 2019). https://doi.org/10.1177/1049731509336986

Laca Arocena, F. (2005). Actitudes y comportamientos en las situaciones de conflicto. Enseñanza e Investigación en Psicología, 10(1), 117-126.

Lara Ros, M. L., Rodríguez Jiménez, T., Martínez González, A. E. y Piqueras, J. A. (2017). Relación entre el bullying y el estado emocional y social en niños de educación primaria. Revista de Psicología Clínica con Niños y Adolescentes, 4(1), 59-64.

Lévy Mangin J. P. (2003). Modelización y análisis con ecuaciones estructurales. Madrid: Prentice Hall.

Lorenzo, L., Ruiz Díaz, A., Toyos, V., Benitez, S. y Santos, L. (2011). Deserción en bachillerato y situación laboral: el caso de tres liceos cercanos a Montevideo. Páginas de Educación, 4(1). http://www.scielo.edu.uy/scielo.php?script=sci_arttext\&pid=S1688-74682011000100005 (Consultado el 27 de septiembre de 2018).

Majata Álvarez, B. R., Flores Palacios, M. G. y García Lara, G. A. (2015). La expresión emocional en la víctima de violencia escolar. Tuxtla Gutiérrez: Facultad de Ciencias Humanas y Sociales de la Universidad de Ciencias y Artes de Chiapas. http://promep.sep.gob. mx/archivospdf/MEMORIAS/Producto2510853.PDF (Consultado el 9 de enero de 2019).

Martínez Ferrer, B., Murgui Pérez, S., Musitu Ochoa, G. y Monreal Gimeno, M. (2008). El rol del apoyo parental, las actitudes hacia la escuela y la autoestima en la violencia escolar en adolescentes. International Journal of Clinical and Health Psychology, 8(3), 679-692.

Menesini, E. y Salmivalli, CH. (2017). Bullying in schools: the state of knowledge and effective interventions. Psychology, Health \& Medicine, 22(1), 240-253. https://doi.org/10.1080/ 13548506.2017.1279740

Mollá López, L., Prado Gascó, V. J. y Martínez Rico, G. (2015). Bullying e inteligencia emocional en niños. Calidad de Vida y Salud, 8(2), 131-149.

Muñoz Abundez, G. (2008). Violencia escolar en México y en otros países: comparaciones a partir de los resultados del Instituto Nacional para la Evaluación de la Educación. Revista Mexicana de Investigación Educativa, 13(39). http://www.scielo.org.mx/scielo. php?script=sci_arttext\&pid=\$1405-66662008000400008 (Consultado el 4 de septiembre de 2018).

Muñoz Prieto, M. M. (2017). Desarrollar la inteligencia emocional para prevenir el acoso escolar. EA, Escuela abierta, 20, 35-46. https://doi.org/https://doi.org/10.29257/EA20.2017.04

Narro Robles, J., Martuscelli Quintana, J. y Barzana García, E. (Coord.). (2012). Plan de diez años para desarrollar el Sistema Educativo Nacional. México: Dirección General de Publicaciones y Fomento Editorial, UNAM. http://www.planeducativonacional.unam. mx (Consultado el 9 de noviembre de 2018).

Narro Robles, J. y Moctezuma Navarro, D. (2012). Analfabetismo en México: una deuda social. Realidad, Datos y Espacio Revista Internacional de Estadística y Geografía, 3(3), 5-17. 
MARIO ALBERTO GONZÁLEZ MEDINA Y DIANA CAROLINA TREVIÑO VILLARREAL VIOLENCIA ESCOLAR EN BACHILLERATO: ALGUNAS ESTRATEGIAS PARA SU PREVENCIÓN DESDE DIFERENTES PERSPECTIVAS

Navarrete Noble, R. E. (2016). En esta escuela, al que no es cábula, lo cabulean. Breve análisis sobre la violencia escolar en México. Boletín Científico de la Escuela Superior de Atotonilco de Tula, 3(5). https://www.uaeh.edu.mx/scige/boletin/atotonilco/n5/e4.html (Consultado el 9 de noviembre de 2018).

Oficina Internacional del Trabajo (2014). El trabajo infantil y el derecho a la educación en México. México: Ediciones Culturales Paidós.

Oliva, H. A. (2015). Matices cronológicos de la violencia escolar en El Salvador. (Apuntes críticos para la prevención del fenómeno). Realidad y Reflexión, 15(42), 11-38. https:// doi.org/10.5377/ryr.v42i0.2815

Organización Internacional del Trabajo (2012). Peligros invisibles del trabajo infantil y adolescente en Paraguay. Asunción: Organización Internacional del Trabajo.

Organización Mundial de la Salud (2014). La prevención de la violencia: evaluación de los resultados de programas de educación para padres. Ginebra: Organización Mundial de la Salud.

Pacheco Salazar, B. (2018). Violencia escolar: la perspectiva de estudiantes y docentes. Revista Electrónica de Investigación Educativa, 20(1), 112-121. https://doi.org/10.24320/ redie.2018.20.1.1523

Pérez Cabaní, M. L., Carretero Torres, M. R. y Juandó Bosch, J. (2001). Afectos, emociones y relaciones en la escuela. Análisis de cinco situaciones cotidianas en educación infantil, primaria y secundaria. Barcelona: GRAÓ.

Pérez García, A. (2016). Bullying en bachillerato: una aproximación a la violencia escolar como fenómeno social. México: Createspace Independent Publishing Platform.

Poujol Galván, G. (2016). Puntos de articulación entre violencia social, exclusión y violencia escolar. Revista Latinoamericana de Estudios Educativos, 46(2), 123-144.

Prieto Quezada, M. T., Carrillo Navarro, J. C. y Jiménez Mora, J. (2005). La violencia escolar. Un estudio en el nivel medio superior. Revista Mexicana de Investigación Educativa, 10(27), 1027-1045.

Quintana Peña, A., Montgomery, W. y Malaver Soto, C. (2009). Modos de afrontamiento y conducta resiliente en adolescentes espectadores de violencia entre pares. Revista de Investigación en Psicología, 12(1), 153-171. http://dx.doi.org/10.15381/rinvp.v12i1.3788

Real Academia Española (2017a). Definición de meta. Madrid: Real Academia Española.

Real Academia Española (2017b). Definición de bien. Madrid: Real Academia Española.

Rodríguez Figueroa, H. M. (2015). La violencia escolar en el bachillerato. Un estudio de caso. Caleidoscopio, 33, 15-43.

Rojas Bravo, J. M. (2013). Clima escolar y tipología docente: la violencia escolar en las prácticas educativas. Cuadernos de Investigación Educativa, 4(19), 87-104.

Romero Bojórquez, L., Utrilla Quiroz, A. y Utrilla Quiroz, V. M. (2014). Las actitudes positivas y negativas de los estudiantes en el aprendizaje de las matemáticas, su impacto en la reprobación y la eficiencia terminal. Ra Ximbai, 10(5), 291-319.

Ruíz Ramírez, R., García Cué, J. L., Ruíz Martínez, F. y Ruíz Martínez, A. (2018). La relación bullying-deserción escolar en bachilleratos rurales. Revista Electrónica de Investigación Educativa, 20(2), 37-45. https://doi.org/10.24320/redie.2018.20.2.1527 
Salcido Cibrián, L. J. y Urías Aguirre, K. M. (2016). Educación emocional en casos de violencia y victimización escolar: propuesta de intervención. Opción, 32 (12), 2088-2102. http:// www.redalyc.org/html/310/31048903057/ (Consultado el 27 de agosto de 2018).

Sánchez, V., Ortega, R. y Menesini, E. (2012). La competencia emocional de agresores y víctimas de bullying. Anales de Psicología, 28(1), 71-82.

Secretaría de Educación del Estado de Nuevo León (2017). Guía para la detección temprana $y$ protocolo de actuación en casos de: abuso sexual infantil, acoso y/o violencia escolar, y maltrato infantil, en las escuelas de educación básica públicas y particulares. Monterrey: Secretaría de Educación del Estado de Nuevo León.

Secretaría de Educación Pública (s.f.). Lo que debes saber. La violencia escolar. México: Secretaría de Educación Pública. http://sep.gob.mx/es/acosoescolar/La_violencia_escolar (Consultado el 1 de octubre de 2018).

Secretaría de Educación Pública (2017). Desarrollar habilidades socioemocionales, una forma de prevenir el abandono escolar en estudiantes de bachillerato. México: Secretaría de Educación Pública. http://www.sems.gob.mx/es_mx/sems/desarrollar-habilidades-socioemocionales-una-forma-de-prevenir-el-abandono-escolar-en-estudiantes-de-bachillerato (Consultado el 5 de octubre de 2018).

Secretaría de Educación Pública y Programa de las Naciones Unidas para el Desarrollo (S.f.). ¿Las emociones pueden ser un obstáculo o una ayuda para el cumplimiento de mis metas? México: Secretaría de Educación Pública y Programa de las Naciones Unidas para el Desarrollo. http://www.construye-t.org.mx/resources/pdf/lecciones/leccion3/3.4._E_ Las_emociones_pueden_ser_un_obstaculo.pdf (Consultado el 9 de enero de 2019).

Secretaría del Trabajo y Previsión Social (2014). El trabajo infantil en México: avances y desafíos. México: Secretaría del Trabajo y Previsión Social. http://www.stps.gob.mx/bp/ gob_mx/librotrabajoinfantil.pdf (Consultado el 19 de septiembre de 2018).

Sierra Varón, C. A. (2010). Violencia escolar. Perfiles psicológicos de agresores y víctimas. Poliantea, 6(10), 53-71.

Toledo, M. I., Guajardo, G., Miranda, CH. y Pardo, I. (2018). Propuesta tríadica para el estudio de la violencia escolar. Cinta moebio, 61, 72-79. https://doi.org/10.4067/S0717$554 X 2017000100072$

United Nations International Children's Emergency Fund (s.f.). Violencia y maltrato. México: United Nations International Children's Emergency Fund. https://www.unicef.org/mexico/ spanish/proteccion_6932.htm (Consultado el 18 de septiembre de 2018).

Usluel, Y. K., Aşkar, P. y Baş, T. (2008). A Structural Equation Model for ICT Usage in Higher Education. Educational Technology \& Society, 11(2), 262-273.

Valdés Cuervo, Á. A., Carlos Martínez, E. A. y Torres Acuña, G. M. (2012). Diferencias en la situación socioeconómica, clima y ajuste familiar de estudiantes con reportes de bullying y sin ellos. Psicología desde el Caribe, 29(3). http://rcientificas.uninorte.edu.co/index. php/psicologia/article/view/3956/9157 (Consultado el 20 de septiembre de 2018).

Vallés Arándiga, A. (2014). Emociones y sentimientos en el acoso escolar. Revista digital EOS Perú, 3(1), 7-17. https://eosperu.net/revista/wp-content/uploads/2015/10/EMOCIONESY-SENTIMIENTOS-EN-EL-ACOSO-ESCOLAR.pdf (Consultado el 9 de enero de 2019). 
MARIO ALBERTO GONZÁLEZ MEDINA Y DIANA CAROLINA TREVIÑO VILLARREAL VIOLENCIA ESCOLAR EN BACHILLERATO: ALGUNAS ESTRATEGIAS PARA

SU PREVENCIÓN DESDE DIFERENTES PERSPECTIVAS

Vitoria Hugo, D. (2006). Metas y emociones cuando se aprende a enseñar ciencias por autorregulación. Revista Electrónica de Motivación y Emoción, 9(22). http://reme.uji.es/ articulos/numero22/article3/nemero\%2022\%20article\%203\%20METAS.pdf (Consultado el 9 de enero de 2018).

Volungis, A. M. y Goodman, K. (2017). School violence prevention: Teachers establishing relationships with students using counseling strategies. SAGE Open, 7(1), 1-11. https:// doi.org/10.1177/2158244017700460.

Zurita Ortega, F. Vilches Aznar, J. M. Martínez Martínez, A. y Castro Sánchez, M. (2015). Influencia de las calificaciones académicas e itinerario curricular sobre conductas violentas. Revista Quadrimestral da Associação Brasileira de Psicologia Escolar e Educacional, SP, 19(3), 503-513. http://dx.doi.org/10.1590/2175-3539/2015/0193885 